\title{
Calculation of Reaction Cross Sections for Producing Br-75 in the Energy Range of 5-40 MeV
}

\author{
H. Salman, B. OruncaK*, R. Ünal \\ Afyon Kocatepe University, Physics Department, Afyonkarahisar, Turkey
}

\begin{abstract}
The isotope of bromine, ${ }^{75} \mathrm{Br}\left(T_{1 / 2}=1.6 \mathrm{~h}\right)$, has a considerable interest in nuclear medicine. The radionuclide ${ }^{75} \mathrm{Br}$ is a positron emitter and is useful in positron emission tomography (PET) for fast metabolic processes. In this study the reaction cross sections were calculated for suggested various production mechanisms. The cross sections of charged particle-induced reactions, leading to ${ }^{75} \mathrm{Br}$, were calculated for ${ }^{76} \mathrm{Se}(\mathrm{p}, 2 \mathrm{n}){ }^{75} \mathrm{Br},{ }^{76} \mathrm{Se}(\mathrm{d}, 3 \mathrm{n}){ }^{75} \mathrm{Br}$, ${ }^{75} \mathrm{As}\left({ }^{3} \mathrm{He}, 3 \mathrm{n}\right){ }^{75} \mathrm{Br}$ and ${ }^{75} \mathrm{As}\left({ }^{4} \mathrm{He}, 4 \mathrm{n}\right){ }^{75} \mathrm{Br}$ using TALYS 1.6. The calculated results are compared with measurement data available in literature. The calculated results indicate that, ${ }^{76} \mathrm{Se}(\mathrm{p}, 2 \mathrm{n}){ }^{75} \mathrm{Br}$ and ${ }^{76} \mathrm{Se}(\mathrm{d}, 3 \mathrm{n}){ }^{75} \mathrm{Br}$ reaction channels have higher cross section values than that of ${ }^{75} \mathrm{As}\left({ }^{3} \mathrm{He}, 3 \mathrm{n}\right){ }^{75} \mathrm{Br}$ reaction channel. Moreover all these reaction channels are in the range of a medium-sized cyclotron.
\end{abstract}

DOI: 10.12693/APhysPolA.128.B-225

PACS: 25.60.Dz

\section{Introduction}

Positron emission tomography (PET) requires positron emitters. Some radioactive bromine isotopes, such as ${ }^{75} \mathrm{Br}$ and ${ }^{76} \mathrm{Br}$, decay with positron emission [1]. Since $\mathrm{C}-\mathrm{Br}$ bond is relatively stronger than the C-I bond [2] and since bromine is an easier replacement for a $\mathrm{H}$ atom in an organic compound [3], bromine is useful in nuclear medicine. However most bromine isotopes have relatively high abundance of $\gamma$-lines above $500 \mathrm{keV}$, which results in diffused images, if only the conventional detection equipment is used. This is why one needs to use the conventional detection equipment in combination with positron detection camera. Bromine has two stable isotopes, ${ }^{79} \mathrm{Br}(50.69 \%)$ and ${ }^{81} \mathrm{Br}(49.31 \%)$. Isotopes ${ }^{70} \mathrm{Br}$ through ${ }^{78} \mathrm{Br}$ decay via electron capture or positron emission. Considering the half-life, isotopes of bromine- $74,75,76$, and 77 (25.4 min, $96.7 \mathrm{~min}, 16.2 \mathrm{~h}$ and $57.036 \mathrm{~h}$ respectively) were found to be suitable in nuclear medicine [4]. Usage of bromine-74 and 76 is out of question because their high positron energy will result in increased exposure of the patient as well as in imaging difficulties [2]. Bromine-77 is also not suitable because its decay mode in $99 \%$ is by electron capture, which is not useful for the positron detection camera [2]. ${ }^{75} \mathrm{Br}$ decays by $71 \%$ positron emission and $29 \%$ electron capture, which makes ${ }^{75} \mathrm{Br}$ useful for positron detection camera [2]. ${ }^{75} \mathrm{Br}$ decays to ${ }^{75} \mathrm{Se}$, which has a 120 days half-life and emits several gamma lines in the 100-300 keV energy range [3]. This contributes to the overall radiation intensity of the ${ }^{75} \mathrm{Br}$-containing radiotracers. The positron end-point energy is $1.7 \mathrm{MeV}$ with several gamma ray lines, with the most prominent being at $286.5 \mathrm{keV}$. In this study, ${ }^{76} \mathrm{Se}(\mathrm{p}, 2 \mathrm{n}){ }^{75} \mathrm{Br},{ }^{76} \mathrm{Se}(\mathrm{d}, 3 \mathrm{n}){ }^{75} \mathrm{Br},{ }^{75} \mathrm{As}\left({ }^{3} \mathrm{He}, 3 \mathrm{n}\right){ }^{75} \mathrm{Br}$

*corresponding author; e-mail: boruncak@aku.edu.tr and ${ }^{75} \mathrm{As}\left({ }^{4} \mathrm{He}, 4 \mathrm{n}\right){ }^{75} \mathrm{Br}$ reaction cross sections were calculated and compared with the experimental data existing in literature.

\section{Calculation methods}

TALYS 1.6 [5] computer code can address the physics of the reaction with default models and parameters. In addition, for more precise calculation, user can customize the basic input. TALYS 1.6 computer code contains such physical models as gamma strength functions, pre-equilibrium models, pre-equilibrium spin distributions, optical model parameters, fission parameters, level density parameters, etc. ${ }^{76} \mathrm{Se}(\mathrm{p}, 2 \mathrm{n}){ }^{75} \mathrm{Br},{ }^{76} \mathrm{Se}(\mathrm{d}, 3 \mathrm{n}){ }^{75} \mathrm{Br}$, ${ }^{75} \mathrm{As}\left({ }^{3} \mathrm{He}, 3 \mathrm{n}\right){ }^{75} \mathrm{Br}$ and ${ }^{75} \mathrm{As}\left({ }^{4} \mathrm{He}, 4 \mathrm{n}\right){ }^{75} \mathrm{Br}$ reaction cross sections have been calculated using several models.

In reaction cross section calculation for ${ }^{76} \mathrm{Se}(\mathrm{p}, 2 \mathrm{n})^{75} \mathrm{Br}$, Brink-Axel Lorentzian gamma-ray strength function model, numerical transition rates with optical model for collision probability pre-equilibrium and microscopic level densities model (Skyrme force) from Goriely's tables were used. ${ }^{76} \mathrm{Se}(\mathrm{d}, 3 \mathrm{n}){ }^{75} \mathrm{Br}$ reaction cross sections were calculated using Brink-Axel Lorentzian gamma-ray strength function model, analytical transition rates with energy-dependent matrix element pre-equilibrium model and constant temperature-Fermi gas level density model. For ${ }^{75} \mathrm{As}\left({ }^{3} \mathrm{He}, 3 \mathrm{n}\right){ }^{75} \mathrm{Br}$ reaction calculation, the KopeckyUhl generalized Lorentzian gamma-ray strength function model and microscopic level densities model (temperature dependent HFB, Gogny force) from Hilaire's combinational tables were used. The pre-equilibrium was disabled and multiple pre-equilibrium was active. ${ }^{75} \mathrm{As}\left({ }^{4} \mathrm{He}, 4 \mathrm{n}\right){ }^{75} \mathrm{Br}$ reaction cross sections were calculated using Brink-Axel Lorentzian gamma-ray strength function model, numerical transition rates with energydependent matrix element pre-equilibrium model and generalized superfluid level density model. 


\section{Results and discussion}

The calculated ${ }^{76} \mathrm{Se}(\mathrm{p}, 2 \mathrm{n}){ }^{75} \mathrm{Br}$ reaction cross sections were compared with the experimental data measured by A.M.J. Paans, et al. [2], Z. Kovacs, et al. [6] and H.E. Hassan, et al. [1]. ${ }^{76} \mathrm{Se}(\mathrm{d}, 3 \mathrm{n}){ }^{75} \mathrm{Br},{ }^{75} \mathrm{As}\left({ }^{3} \mathrm{He}, 3 \mathrm{n}\right){ }^{75} \mathrm{Br}$ and ${ }^{75} \mathrm{As}(\alpha, 4 \mathrm{n}){ }^{75} \mathrm{Br}$ reaction cross sections were also calculated and compared with the experimental data measured by A.M.J. Paans et al. [2].

Calculations yielded cross section values of over $400 \mathrm{mb}$ for ${ }^{76} \mathrm{Se}(\mathrm{p}, 2 \mathrm{n}){ }^{75} \mathrm{Br}$ reaction channel in the 22-26 MeV energy range (see Fig. 1) and over $350 \mathrm{mb}$ for ${ }^{76} \mathrm{Se}(\mathrm{d}, 3 \mathrm{n}){ }^{75} \mathrm{Br}$ reaction channel in the $28-34 \mathrm{MeV}$ energy range (see Fig. 2). The calculated reaction cross sections for ${ }^{75} \mathrm{As}\left({ }^{3} \mathrm{He}, 3 \mathrm{n}\right){ }^{75} \mathrm{Br}$ are over $200 \mathrm{mb}$ in the 25-30 MeV energy range (see Fig. 3). There are discrepancies between the experimental data and the calculated cross sections. The calculated reaction cross sections for ${ }^{75} \mathrm{As}(\alpha, 4 \mathrm{n}){ }^{75} \mathrm{Br}$ are in agreement with experimental results up to incident energy of $60 \mathrm{MeV}$ (see Fig. 4). However presented calculation results deviate well below the experimental data for incident energy above $60 \mathrm{MeV}$.

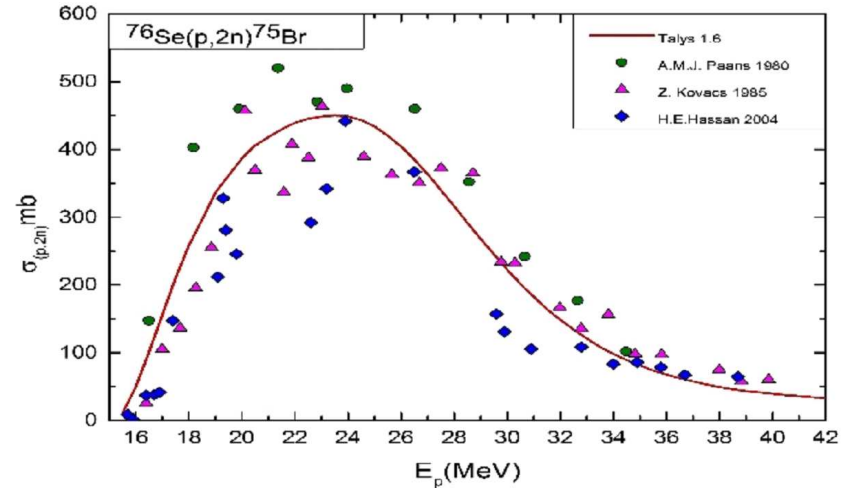

Fig. 1. Comparison of the calculated Talys 1.6 ${ }^{76} \mathrm{Se}(\mathrm{p}, 2 \mathrm{n}){ }^{75} \mathrm{Br}$ reaction cross section data with data from $[2]$.

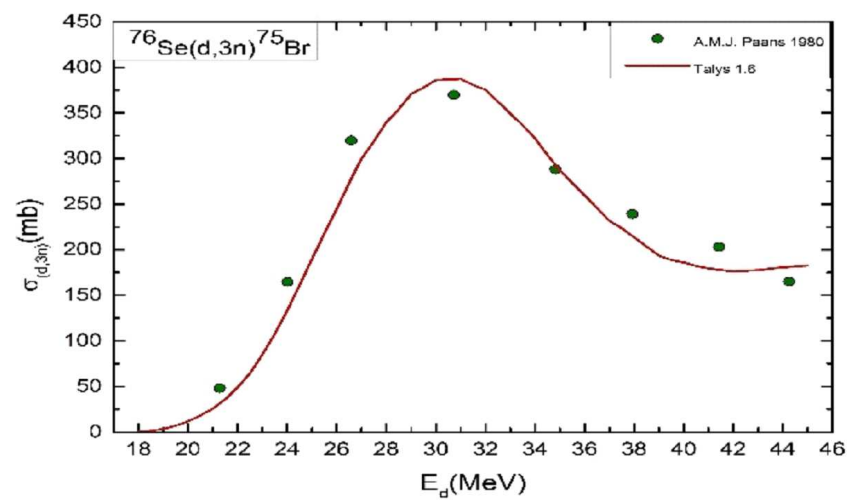

Fig. 2. Comparison of the calculated Talys 1.6 ${ }^{76} \mathrm{Se}(\mathrm{d}, 3 \mathrm{n})^{75} \mathrm{Br}$ reaction cross section data with data from [2].

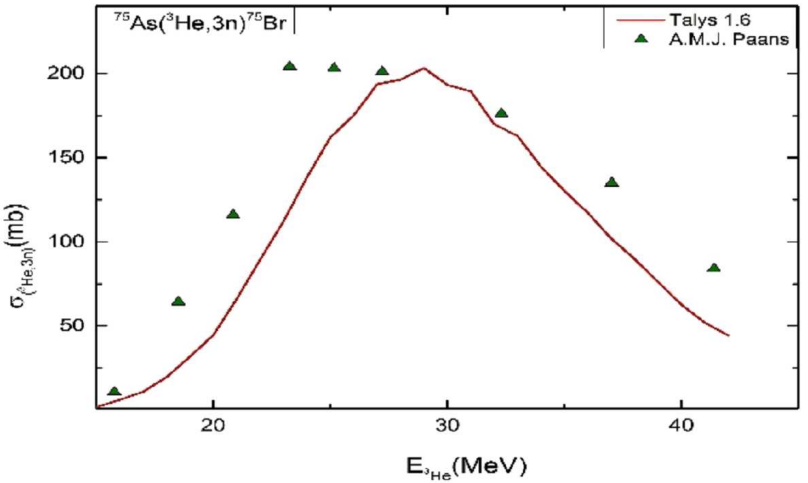

Fig. 3. Comparison of the calculated Talys 1.6 ${ }^{74} \mathrm{As}\left({ }^{3} \mathrm{He}, 3 \mathrm{n}\right){ }^{75} \mathrm{Br}$ reaction cross section data with data from $[2,6,1]$.

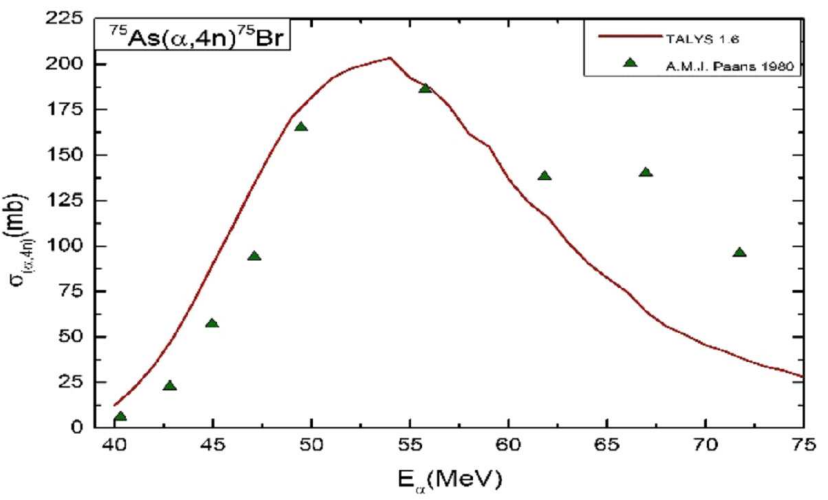

Fig. 4. Comparison of the calculated Talys 1.6 ${ }^{74} \mathrm{As}(\alpha, 4 \mathrm{n}){ }^{75} \mathrm{Br}$ reaction cross section data with data from [2].

\section{Conclusions}

The presented calculations indicate that favorable production routes for bromine- 75 are the ${ }^{76} \mathrm{Se}(\mathrm{p}, 2 \mathrm{n})^{75} \mathrm{Br}$ and ${ }^{76} \mathrm{Se}(\mathrm{d}, 3 \mathrm{n}){ }^{75} \mathrm{Br}$ reactions. The energy range for such high cross sections is within the limit of a medium-sized cyclotron. Even though presented calculation and experimental data is in agreement for ${ }^{75} \mathrm{As}\left({ }^{3} \mathrm{He}, 3 \mathrm{n}\right){ }^{75} \mathrm{Br}$ reaction cross sections, the total cross section is well below the ones of the mentioned above reaction channels. The cross sections for ${ }^{75} \mathrm{As}(\alpha, 4 \mathrm{n}){ }^{75} \mathrm{Br}$ reaction are comparable with ${ }^{75} \mathrm{As}\left({ }^{3} \mathrm{He}, 3 \mathrm{n}\right){ }^{75} \mathrm{Br}$ reaction cross sections, however this reaction requires higher incident energy, which is beyond the scope of medium-sized cyclotron. Further studies of ${ }^{75} \mathrm{As}\left({ }^{3} \mathrm{He}, 3 \mathrm{n}\right){ }^{75} \mathrm{Br}$ reaction are recommended to resolve the presented discrepancies. 


\section{References}

[1] H.E. Hassan, S.M. Qaim, Yu. Shubin, A. Azzam, M. Morsy, H.H. Coenen, Appl. Radiat. Isotopes 60, 899 (2004).

[2] A.M.J. Paans, J. Welleweerd, W. Vaalburg, S. Reiffers, M.G. Woldring, Int. J. Appl. Radiat. Is. 31, 267 (1980).

[3] I. Spahn, M.M. Shehata, S. Spellerberg, B. Scholten, H.H. Coenen, S.M. Qaim, J. Korean Phys. Soc. 59, 1983 (2011).

[4] International Atomic Energy Agency, Cyclotron Produced Radionuclides: Physicsal Characteristics and Production Methods, Technical Reports Series No. 468 (2009) Vienna.
[5] A.J. Koning, S. Hilaire, M.C. Duijvestijin, Proceedings of the International Conference on Nuclear Data for Science and Technology, Eds. O. Bersillon, F. Gunsing, E. Bauge, R. Jacqmin, S. Leray, EDP Sciences, Nice, France 2008, p. 211.

[6] Z. Kovacs, G. Blessing, S.M. Qaim, G. Stöcklin, Int. J. Appl. Radiat. Is. 36, 635 (1985).

[7] www-nds.iaea.org/exfor/exfor.htm (15 April 2014). 